\title{
Leiomyosarcoma of the Rectum: \\ The First Report of Effective Treatment with Endoscopic Submucosal Dissection
}

Eduardo Guimarães de Moura, MD, PhD

Gastrointestinal Endoscopy Unit, Department of Gastroenterology, University of São Paulo Medical School, São Paulo, Brazil.

Vitor Ottoboni Brunaldi, MD

Gastrointestinal Endoscopy Unit, Department of Gastroenterology, University of São Paulo Medical School, São Paulo, Brazil.

E-mail: vitor.brunaldi@usp.br; vbrunaldi@gmail.com

Flavio Hiroshi Ananias Morita, MD

Gastrointestinal Endoscopy Unit, Department of Gastroenterology, University of São Paulo Medical School, São Paulo, Brazil.

Diogo Turiani de Moura, MD

Gastrointestinal Endoscopy Unit, Department of Gastroenterology, University of São Paulo Medical School, São Paulo, Brazil.

\section{Eduardo Turiani de Moura, MD}

Gastrointestinal Endoscopy Unit, Department of Gastroenterology, University of São Paulo Medical School, São Paulo, Brazil.

\section{Humberto Kishi, MD}

Diagnóstika Pathology Unit, Department of Gastrointestinal Pathology, São Paulo, Brazil.

Elisa Ryoka Baba, MD

Gastrointestinal Endoscopy Unit, Department of Gastroenterology, University of São Paulo Medical School, São Paulo, Brazil.

(c) Mary Ann Liebert, Inc. DOI: 10.1089/vor.2017.0461
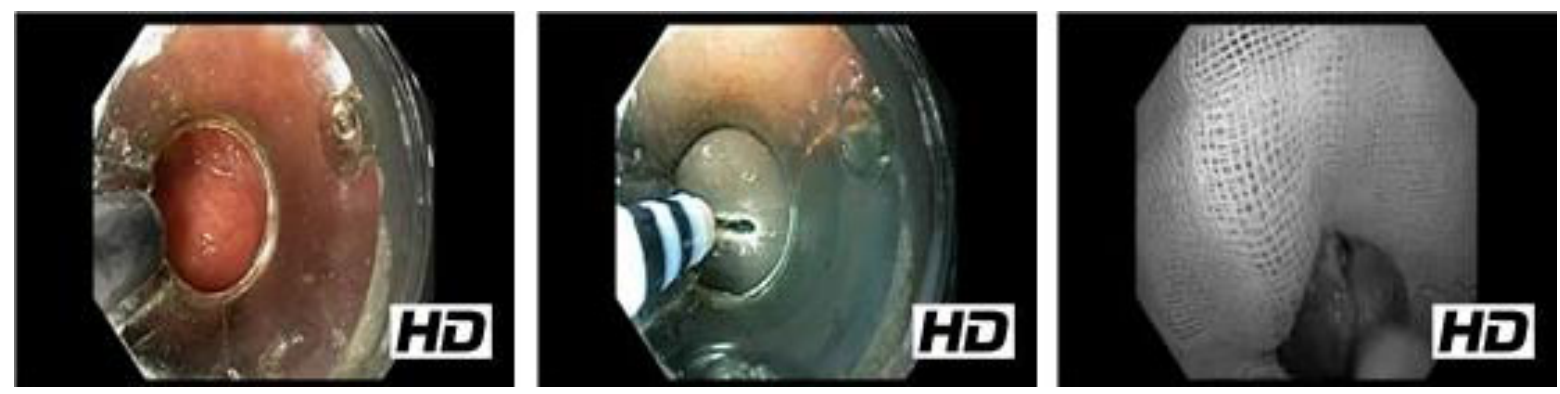

\section{Abstract}

Background: Rectal leiomyosarcoma (LMS) is an extremely rare smooth muscle tumor accounting for $<0.1 \%$ of all rectal neoplasms. ${ }^{1}$ Because of its rarity, there is no current consensus on the adequate therapeutic strategy. ${ }^{2}$ This video demonstrates an LMS of the rectum treated effectively with endoscopic submucosal dissection (ESD).

Methods/Results: A 68-year-old male patient underwent a screening colonoscopy that identified a $3 \mathrm{~cm}$ submucosal tumor in the lower rectum, $5 \mathrm{~cm}$ above the anal verge. Endoscopic ultrasonography 
showed a hypoechoic, homogenous, well-defined mass arising from the deep mucosa layer. Tissue sample was obtained with fine-needle aspiration that showed mesenchymal myoid cells that did not stain for CD117, chromogranin, or synaptophysin. Smooth muscle actin (SMA) was focally positive. Hence, the presumed diagnosis was a submucosal leiomyoma. An ESD was effectively performed with en bloc resection and no immediate or late complications. The total length of procedure was 225 minutes. Histology showed a cellular spindle cell neoplasm having variable eosinophilic cytoplasm and mild atypical tapering or more cigar-shaped nuclei with scattered mitotic figures. The resection specimen had tumor-free margins despite an infiltrative growth pattern. Immunohistochemistry showed diffusely positive staining for SMA and desmin, while negative for S-100 protein, GFAP, and SOX10. The Ki-67 score was $<2 \%$. The findings were compatible with a low-grade LMS of the rectum. Based on the limited evidence available in the literature, a multidisciplinary team decided for an additional full-thickness resection with transanal endoscopic microsurgery (TEM). The new specimen from the TEM was tumor free. The patient is currently on 6-month follow-up, with no signs of local recurrence or metastasis.

Discussion/Conclusion: ESD may be an adequate therapy for LMS of the rectum in selected cases. Further cases need to be performed using this technique and long-term follow-up is required for all patients undergoing this technique.

No competing financial interests exist.

Runtime of video: 6 mins 48 secs

Keywords: leiomyosarcoma, endoscopic submucosal dissection, rectal neoplasms, endoscopy

\section{Cite this video}

Eduardo Guimarães de Moura, Vitor Ottoboni Brunaldi, Flavio Hiroshi Ananias Morita, Diogo Turiani de Moura, Eduardo Turiani de Moura, Humberto Kishi, Elisa Ryoka Baba, Leiomyosarcoma of the Rectum: The First Report of Effective Treatment with Endoscopic Submucosal Dissection, Videoscopy. 2017, DOI: 10.1089/vor.2017.0461.

\section{References}

1. Sahli N, Khmou M, Khalil J, Elmajjaoui S, El Khannoussi B, Kebdani T, Elkacemi H, Benjaafar N. Unusual evolution of leiomyosarcoma of the rectum: A case report and review of the literature. J Med Case Rep 2016;10:249.

2. Hoshino N, Hida K, Kawada K, Sakurai T, Sakai Y. Transanal total mesorectal excision for a large leiomyosarcoma at the lower rectum: A case report and literature review. Surg Case Rep 2017;3:13.

Original Publication Date: 2017 